\title{
Technique, risks, and true impact of routine preoperative endoscopic evaluation of morbidly obese patients undergoing bariatric surgery
}

\author{
Jean-Marc Dumonceau
}

Published online: 17 June 2011

(C) Springer Science+Business Media, LLC 2011

In their report on upper digestive endoscopy prior to bariatric surgery, Küper et al. [1] state that "endoscopy can be performed safely, but anesthesiological support is strongly recommended in patients with critical condition." The authors did not define critical conditions, but one of these is likely sleep apnea syndrome because the incidence of hypoxemia requiring emergency bronchoscopy was significantly higher in patients affected with this syndrome compared to those who were not $[2 / 14$ (14\%) vs. 0/51, respectively; $p<0.05$ (two-tailed Fisher exact test)]. Also, I think that a procedure that resulted in extremely severe hypoxemia $\left(\mathrm{SaO}_{2}<60 \%\right)$ and required emergency bronchoscopy for intratracheal $\mathrm{O}_{2}$ insufflation in $3 \%$ of patients can hardly be regarded as safe. According to a recent survey [2], approximately 350,000 bariatric surgery procedures were performed in 2008 in 36 nations; systematic preoperative endoscopy in these patients (as recommended by the authors) in the conditions described by Küper et al. would have put 10,000 persons at risk of iatrogenic complications.

A useful alternative to endoscopy under sedation in these patients is unsedated transnasal esogastroduodenoscopy. This technique may be performed using a pediatric endoscope, can be learned quickly [3], and has a patient tolerance equivalent to that of sedated esogastroduodenoscopy [4]. It was successfully used without complication in a similar pilot study of 25 morbidly obese patients, of whom $68 \%$ had obstructive sleep apnea [5]. This technique also precludes the increased need in time and resources that

J.-M. Dumonceau ( $\square)$

Service of Gastroenterology and Hepatology,

Geneva University Hospitals, Rue Gabrielle-Perret Gentil 4,

1211 Geneva 14, Switzerland

e-mail: jmdumonceau@hotmail.com is mentioned by the authors for sedated endoscopy in morbidly obese compared to unselected patients.

The authors also state that "upper gastrointestinal endoscopy should be performed in all patients in whom bariatric procedures of any sort are planned." This was motivated by the high incidence of pathological findings that they reported at endoscopy (80\%), but few details were provided. As mentioned by the authors, the most frequent finding (gastritis in $36 \%$ of patients) had no impact on patient management. The relevance of other frequent findings is debatable (hiatal hernia, 27\%; reflux esophagitis, $12 \%$ ). It would be interesting to know how the authors explain (1) the unusually high incidence of gastric peptic ulcers ( $23 \%$ of patients; what was the size of the ulcers and did patients take nonsteroidal anti-inflammatory drugs?), (2) if gastric polyps (9\% of patients) were attributable to acquired fundic gland polyposis related to chronic use of proton-pump inhibitors (requiring no specific management) or were associated with previously undiagnosed congenital conditions, and (3) the final diagnosis and management in patients with submucosal tumors (4.3\% of patients).

When reviewing in detail our findings of preoperative esogastroduodenoscopy in 468 bariatric patients, we found that the true impact of endoscopy in asymptomatic patients was extremely limited and that it even triggered unnecessary workup in some cases [6]. As we found that abnormal findings at endoscopy were highly correlated with Helicobacter pylori status, we have proposed, for asymptomatic patients who are to undergo bariatric surgery, to systematically administer proton-pump inhibitors and to eradicate H. pylori in patients found to be positive using a noninvasive test.

Disclosure J.-M. Dumonceau has no conflicts of interest or financial ties to disclose. 


\section{References}

1. Küper MA, Kratt T, Kramer KM et al (2010) Effort, safety, and findings of routine preoperative endoscopic evaluation of morbidly obese patients undergoing bariatric surgery. Surg Endosc 24:1996-2001

2. Buchwald H, Oien DM (2009) Metabolic/bariatric surgery worldwide 2008. Obes Surg 19:1605-1611

3. Maffei M, Dumortier J, Dumonceau J-M (2008) Self-training in unsedated transnasal EGD by endoscopists competent in standard peroral EGD: prospective assessment of the learning curve. Gastrointest Endosc 67:410-418
4. Stroppa I, Grasso E, Paoluzi OA et al (2008) Unsedated transnasal versus transoral sedated upper gastrointestinal endoscopy: a oneseries prospective study on safety and patient acceptability. Dig Liver Dis 40:767-775

5. Alami RS, Schuster R, Friedland S et al (2007) Transnasal smallcaliber esophagogastroduodenoscopy for preoperative evaluation of the high-risk morbidly obese patient. Surg Endosc 21:758-760

6. Azagury D, Dumonceau J-M, Morel P, Chassot G, Huber O (2006) Preoperative work-up in asymptomatic patients undergoing Rouxen-Y gastric bypass: is endoscopy mandatory? Obes Surg 16:1304-1311 